\title{
BER Based Performance Evaluation by Pulse Shaping in OFDM
}

\author{
D. K. Sharma ${ }^{1}$, A. Mishra ${ }^{2}$ and Rajiv Saxena ${ }^{3}$ \\ ${ }^{1}$ Ujjain Engineering College, \\ Ujjain, INDIA \\ ${ }^{2}$ Madhav Institute of Technology \& Science, Gwalior, INDIA \\ ${ }^{3}$ Jaypee University of Engineering \& Technology, Guna, INDIA \\ dilip_sharma1172@yahoo.com ${ }^{1}$,drabhaymishra@yahoo.com ${ }^{2}$, \\ rajiv.saxena@jiet.ac.in
}

\begin{abstract}
The next generation wireless communication system requires a higher standard in order to provide the customers with high quality of services they demanded. Orthogonal Frequency Division Multiplexing (OFDM) is an attractive modulation method and is a strong candidate for the modulation technique of future wireless systems which can be implemented easily. In this paper an effort is made to analyze how well an OFDM system will perform when transmitted over an Additive White Gaussian Noise (AWGN) channel in terms of Bit Error Rate (BER), it has also been observed that pulse shaping the channels present in an OFDM system promises to improve the overall performance of the system in terms of the BER of the received signals, different window function like Rectangular, Blackman, Gaussian, Hamming, Hanning and Kaiser and some of the self modified pulse shapes have been used to study the BER performance due to any one of the pulse shapes.
\end{abstract}

\section{Keywords: AWGN, ICI, OFDM, BIT ERROR RATE}

\section{Introduction}

High Bandwidth efficiency and high bit error rate are the major requirements of modern communication systems to cater to the needs for a variety of new and high quality services developed and delivered to the society [1]. The effect of multipath delay, spread and fading of signals in the wireless environment is usually unavoidable $[2,3]$. Extreme fading of the signal amplitude and Inter Symbol Interference (ISI) introduced during the transmission through the conventional channel and the frequency selectivity of the channel appearing at the receiver side $[4,5]$ are responsible for a high probability of errors and reduced overall performance of the system. Several methods like adaptive equalization and channel coding have been developed to reduce the above effect $[6,7]$.

The orthogonal Frequency division multiplexing (OFDM) a multi carrier system can be thought of as a solution and is used in several applications such as asymmetric digital subscriber lines (ADSL), a system that makes high bit-rates possible over twisted-pair copper wires [8, 9], it has also been accepted for several wireless LAN standards, as well as a number of mobile multimedia applications $[1,10]$ and protocols like IEEE 802.11a standard, terrestrial digital video broadcasting (DVB-T) and digital audio broadcasting (DAB) [11]. The purpose of this paper is to investigate how OFDM performs in an Additive White Gaussian Noise (AWGN) channel for different window function, like Rectangular, Blackman, Gaussian, Hamming, Hanning and Kaiser. In this channel only one path between the transmitter and the receiver exists and only a constant attenuation and noise is considered.

In this paper, we define the simulation methodology of OFDM in section 2 and simulate the OFDM system using Matlab. The mathematical model and theoretically simulated constellation diagrams for various pulse shapes are presented and a procedure for symbol 
error rate is derived and finally a BER simulation of OFDM for various window functions is presented.

\section{Simulation of OFDM System}

A simplified version of block diagram for an OFDM transmitter and receiver is shown in Figure-1.

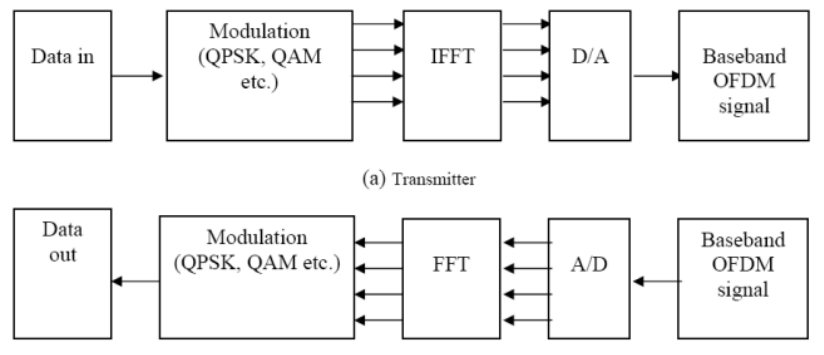

(b) Receiver

\section{Figure 1. Transmitter and Receiver of OFDM}

The OFDM signal generated by the system in Figure-1 is at base band; in order to generate a radio frequency (RF) signal at the desired transmit frequency, filtering and mixing is required. OFDM allows for a high spectral efficiency as the carrier power and modulation technique can be individually controlled for each carrier. However in broadcast systems these are fixed due to the one-way communication [12].

\section{Simulation Methodology}

A 4-QAM symbols with 1705 carrier is simulated and a 4096 point IFFT is applied to it, the generated carrier is then modulated by a pulse shape $g(t)$. The so obtained output is in turn applied to a low pass filter for obtaining a continuous time signal which is then multiplied to the carrier wave generating a signal $x(t)[13,14]$.

\section{Mathematical Model of OFDM}

The expression for an OFDM symbol at $\mathrm{t}=t_{s}$ is given as:

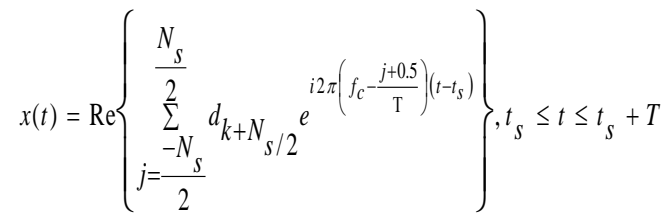

$$
\begin{aligned}
& x(t)=0, t<t_{s} \wedge t>t_{S}+T \ldots \ldots \ldots \ldots \ldots \ldots(1)
\end{aligned}
$$

Where $d_{k}=$ complex modulation symbols, $\mathrm{N}_{S}=$ number of sub carriers, $T=$ symbol duration, $f_{c}=$ carrier frequency.

The above "(1)" is also expressed [12] as:

$$
x(t)=\operatorname{Re}\left\{e^{j 2 \pi f_{c} t} \sum_{m=0}^{\infty} \sum_{l=0}^{67} \sum_{k=k_{\min }}^{k_{\max }} C_{m, l, k} . \psi_{m, l, k}(t)\right\} \ldots .(2)
$$

Where 


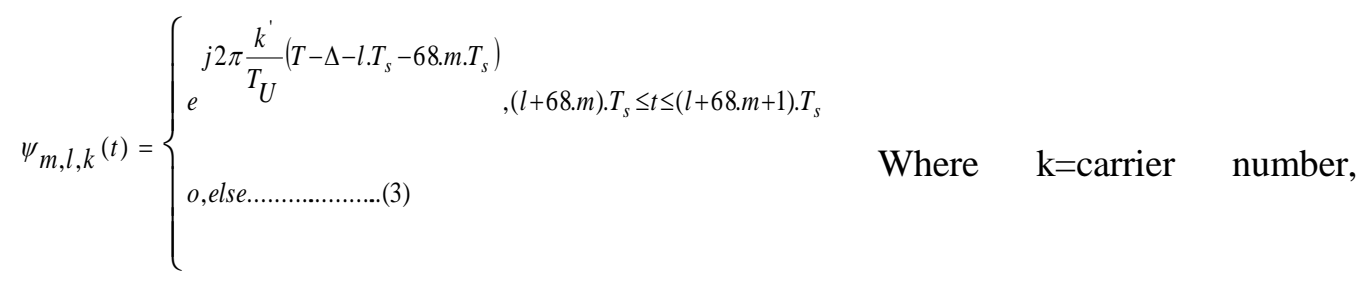

$\mathrm{l}=\mathrm{OFDM}$ symbol number, $\mathrm{m}=$ transmission frame number, $\mathrm{K}=$ number of transmitted carriers, $\mathrm{T}_{S}=$ symbol duration, $\mathrm{T}_{U}=$ inverse of the carrier spacing, $\Delta=$ duration of the guard interval, $f_{c}=$ central frequency of the radio frequency $(\mathrm{RF})$ signal, $\mathrm{k}^{\prime}=$ carrier index relative to the center $\left(k^{\prime}=k-\left(K_{\max }+K_{\min }\right) / 2\right)$ frequency, $C_{m, i, k}=$ complex symbol for the carrier $\mathrm{K}$ of the data symbol frame from number $0,1,2,3 \ldots .67$ in frame number $\mathrm{m}$. The guard interval between the consecutive symbols of OFDM can also reduce the effect of ISI, thus the size of the guard interval can also be a parameter for reducing the ICI and thus wavering the requirement of equalization at the receiving end.

\section{A. OFDM Pulse Shaping}

The pulse shaped OFDM for complex envelope of one radio frequency $N$-sub carrier is given as $[15,16]$

$$
x(t)=e^{i 2 \pi f_{c} t} \sum_{k=0}^{\mathrm{N}-1} d_{k} \sqrt{g(t)} e^{i 2 \pi f_{k} t}
$$

where, $i^{2}=-1, f_{c}$ is the carrier frequency, $f_{k}$ is the sub carrier frequency of the $k^{\text {th }}$ sub carrier, and $d_{k}, k=0,1,2, \ldots, \mathrm{N}-1$, is the complex modulation symbol transmitted on the $k^{\text {th }}$ sub carrier assuming $d_{k}$ has zero mean and normalized average symbol energy and further assuming that the modulation (data) symbols are uncorrelated and are given as

$$
\mathrm{E}\left[d_{k} d_{k}^{*}\right]=\left\{\begin{array}{l}
1, k=m \\
0, k \neq m
\end{array} .\right.
$$

where $d_{k}^{*}$ denotes the complex conjugate of $d_{k}$. The band limited pulse-shaping function is denoted by $g(t)$, but

$$
f_{k}-f_{m}=\frac{k-m}{\mathrm{~T}} \ldots . .(6)
$$

Table 1. Types of Pulses Shaping Function

\begin{tabular}{|c|l|l|}
\hline $\begin{array}{c}\text { Sr } \\
\mathbf{N} \\
\mathbf{o}\end{array}$ & Types of Pulses & Expression of Windows Function \\
\hline $\mathbf{1}$ & Rectangle Pulse & $g(n)=\left\{\begin{array}{l}1, n=1,2,3, \ldots ., M \\
0, \text { otherwise }\end{array}\right.$ \\
\hline $\mathbf{2}$ & $\begin{array}{c}\text { Blackman } \\
\text { pulse }\end{array}$ & $g(n)=0 \cdot 42-0 \cdot 5 \cos \left(\frac{2 \pi n}{\mathrm{M}-1}\right)$ \\
& $+0 \cdot 08 \cos (4 \pi n / \mathrm{M}-1)$. \\
\hline
\end{tabular}




\begin{tabular}{|c|c|c|}
\hline 3 & $\begin{array}{l}\text { Gaussian } \\
\text { pulse }\end{array}$ & $\begin{array}{l}g(n)=\exp \left(-\frac{1}{2}[2 \beta n / \mathrm{N}]^{2}\right) \\
\text { for } 0 \leq\langle n\rangle \leq \frac{\mathrm{N}}{2}\end{array}$ \\
\hline 4 & Hamming pulse & $g(n)=0 \cdot 54-0 \cdot 46 \cos (2 \pi n / M-1)$ \\
\hline 5 & $\begin{array}{l}\text { Hanning } \\
\text { Pulse }\end{array}$ & $\mathrm{g}(\mathrm{n})=\frac{1}{2}(1-\cos 2 \pi n / \mathrm{M}-1)$ \\
\hline 6 & Kaiser pulse & $\begin{array}{l}\mathrm{g}(\mathrm{n})=\frac{I_{0}\{\beta \sqrt{I-(n / M)}\}}{I_{0}(\beta)},-M \leq n \leq M . \text { Where } \beta \\
\text { is an adjustable parameter and } I_{0}(u) \text { is modified } \\
\text { Zeroth-order Bessel function. }\end{array}$ \\
\hline
\end{tabular}

Is taken to ensure the sub carrier orthogonality [3], which can be defined as

$$
\int_{-\infty}^{\infty} g(t) e^{i 2 \pi\left(f_{k}-f_{m}\right) t} d t=\left\{\begin{array}{l}
1, k=m \\
0, k \neq m
\end{array} \ldots . .(7)\right.
$$

Where $\frac{1}{\mathrm{~T}}$ is the minimum sub carrier frequency spacing required. The Fourier transform for the pulse $g(t)$ should have spectral nulls at the frequencies $\pm \frac{1}{T}, \pm \frac{2}{T}, \ldots$. to ensure sub carrier orthogonality.

Modulation of the OFDM signal [13] is done by applying the pulse $\mathrm{g}(\mathrm{t})$, and an exhaustive analysis has been carried out by varying the pulse shape $\mathrm{g}(\mathrm{t})$ of discrete version $\mathrm{g}(\mathrm{n})$ in order to search for a better combination off the bit error rate and modulating pulse shape as given in Table-I.

\section{B. Constellation Diagrams}

The alphabets used for a 4-QAM $\alpha_{4-Q A M}=\{ \pm 1 \pm 1 j\}$ are as shown in Figure-2. Constellation diagrams [9] are used to graphically represent the quality and distortion of a pulse shaped OFDM signal.

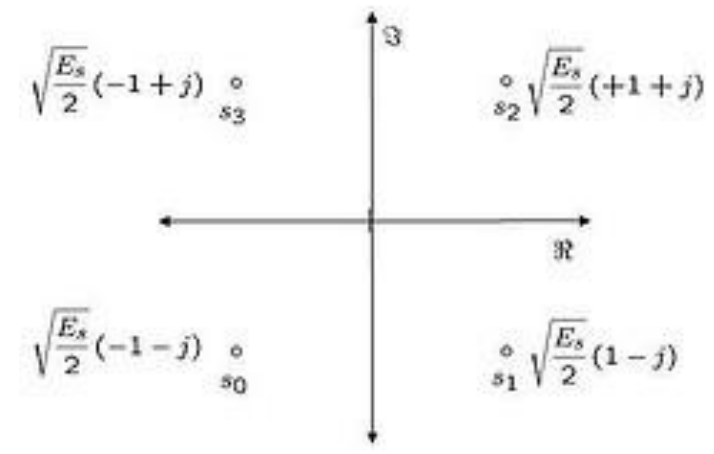

Figure 2. Orginal Constallarion Plot for 4-QAM 


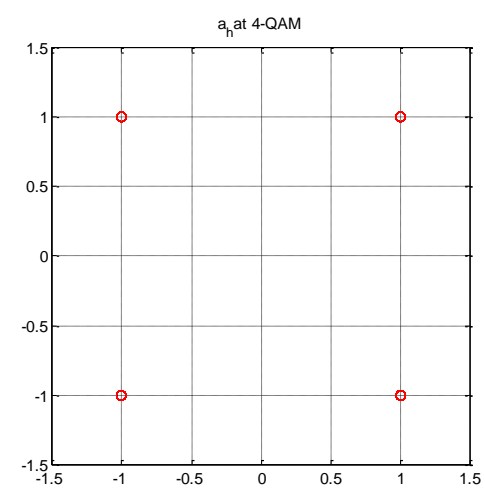

Figure 3. Simulated of Original 4-QAM Constellation

There is always a combination of modulation errors that occurs and is difficult to separate and identify. The original constellation and the received constellations for SNR value of 2, 6, $12 \mathrm{~dB}$ of OFDM are simulated and presented in Figure- 3 and Figure-4 to Figure-6.

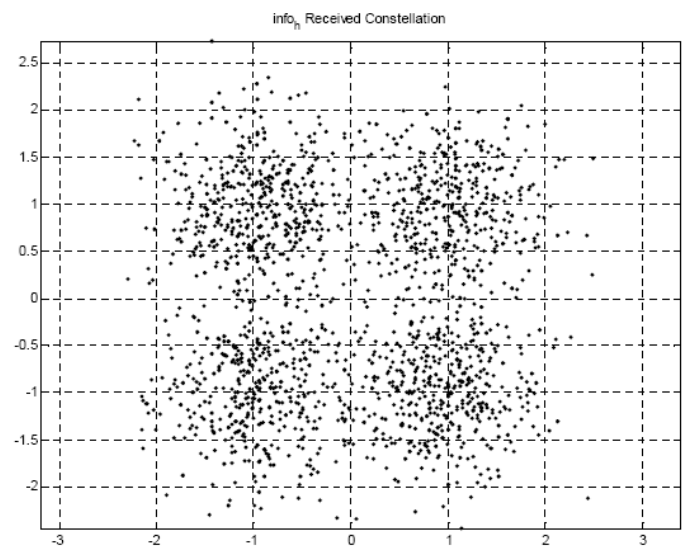

Figure 4. Simulated of Received 4-QAM Constellation for SNR=2dB

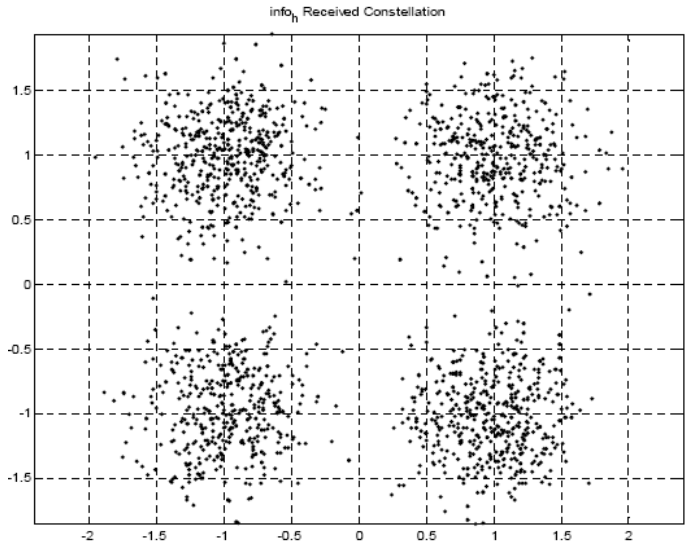

Figure 5. Simulated of Received 4-QAM Constellation for SNR=6dB 


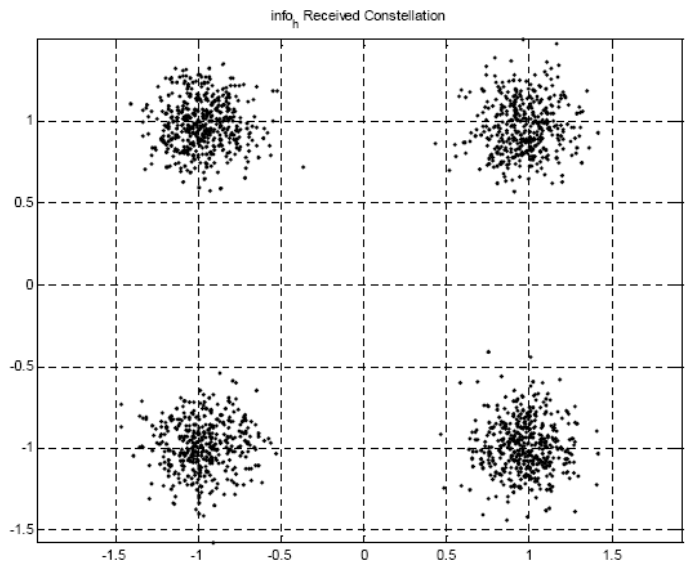

Figure 6. Simulated of Received 4-QAM Constellation for SNR=12dB

An AWGN channel is considered, for simulation the sampled signal for the $k^{\text {th }}$ sub channel. After the receiver Fast Fourier transforms (FFT) processing is written as [16], [17]

$$
r_{k}=d_{k} c_{0}+\sum_{l=0, l \neq k}^{\mathrm{N}-1} d_{l} c_{l-k}+N_{k}, k=0,1, \ldots, \mathrm{N}-1 . .
$$

Where $N_{k}$ Gaussian random variables (RVs) with zero mean and variance $\sigma^{2}$ for real and imaginary components, $d_{k}$ denote the data symbol for the $k^{\text {th }}$ sub carrier where $\mathrm{N}$ is the number of sub carriers.

\section{Effect on ICI}

The second term in "(8)" represents the ICI caused by the frequency offset (FO). The $c_{K}$ sequence (ICI coefficients) depends on the FO and is given by [18]

$$
c_{k}=\frac{\sin \pi(k+\tau)}{\mathrm{N} \sin \frac{\pi}{\mathrm{N}}(k+\tau)} e^{\left[i \pi\left(1-\frac{1}{\mathrm{~N}}\right)(k+\tau)\right]} .
$$

where $\tau$ is the normalized frequency offset which is the ratio between the FO and the adjacent sub carrier spacing. For zero frequency offset, $c_{K}$ reduces to the unit impulse sequence. The variance of the signal constellation, $\mathrm{E}\left|d_{k}\right|^{2}$, will be independent of $\mathrm{k}$ if all sub carriers use the same modulation format, which is the normal case. The variance of the ICI on the $k^{\text {th }}$ sub carrier can be given by

$$
\sigma_{I C I}^{2}=\sum_{l=0, l \neq k}^{\mathrm{N}-1}\left|c_{l-k}\right|^{2}
$$

For M-ary signaling, $d_{k}$ is equally likely to assuming one out of $\mathrm{M}$ levels. The receiver receives it and checks the errors. The simulation is based on multiple signal-tonoise ratios (SNR).

\section{COMPUTING THE PROBABILITY OF ERROR}

The theoretical probability of symbol error for rectangular QAM constellation is given in [8], [18] as follows:

$$
\mathrm{P}_{\mathrm{M}}=1-\left(1-\mathrm{P}_{\sqrt{\mathrm{M}}}\right)^{2}
$$


Where $\mathrm{P}_{\sqrt{\mathrm{M}}}=2\left(1-\frac{1}{\sqrt{\mathrm{M}}}\right) \cdot Q\left(\sqrt{\frac{3 \cdot \mathrm{E}_{a v}}{(\mathrm{M}-1) \cdot \mathrm{N}_{o}}}\right) \ldots \ldots . .(12) \quad$ Here $\mathrm{E}_{a v}$ is the average energy per bit; $\mathrm{M}=2^{k}$ represents the number of levels and $k$ is the number of bits per symbol. Equations (11) and (12) are for the case of $k$ even. For $k$ odd, there is no exact result. However, the symbol-error probability is upper bounded [19] as

$$
\mathrm{P}_{\mathrm{M}} \leq 4 Q\left(\sqrt{\frac{3 k \mathrm{E}_{a v}}{(\mathrm{M}-1) \cdot \mathrm{N}_{o}}}\right) \ldots \ldots . .(13)
$$

The theoretical curve was generated using "(13)", without the scaling factor (i.e. only using the Q-function without the 4 in front), although for $k$ even (i.e. $k=2$ for 4-QAM) [9].

The BER simulation of OFDM systems with pulse shapes have been carried out and are plotted in Figure-7 to Figure-12 for different window function using Rectangular, Blackman, Gaussian, Hamming, Hanning and Kaiser windows.

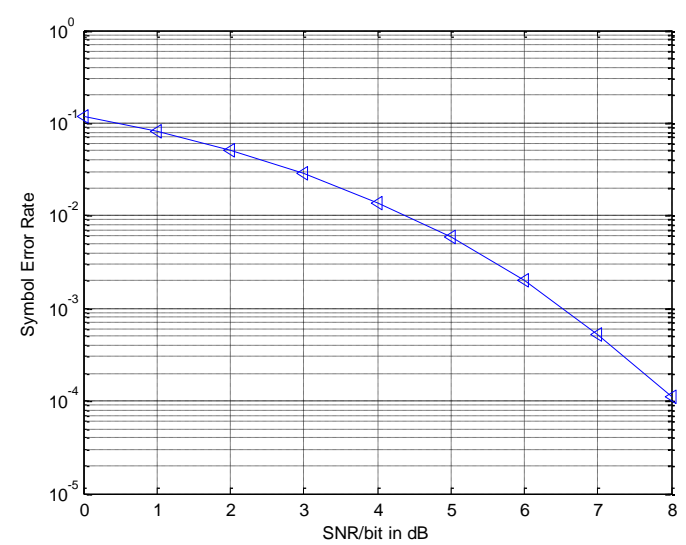

Figure 7. Symblol error rate for Rectangular pulse.

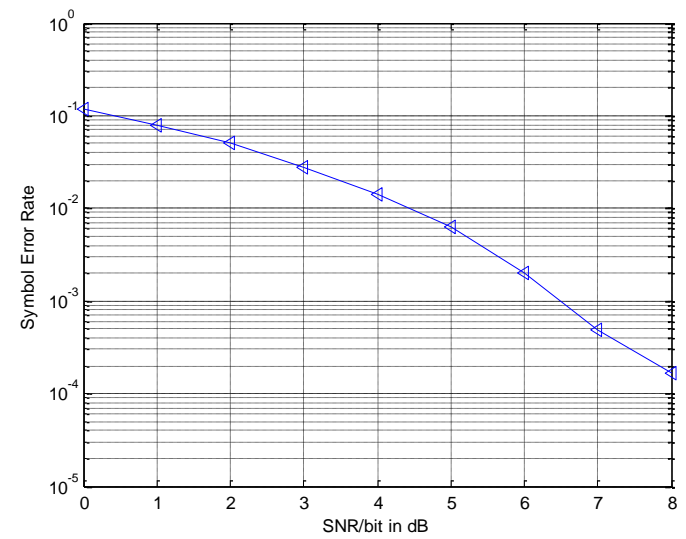

Figure 8. Symblol error rate for Blackman pulse. 
International Journal of Signal Processing, Image Processing and Pattern Recognition Vol.8, No.6 (2015)

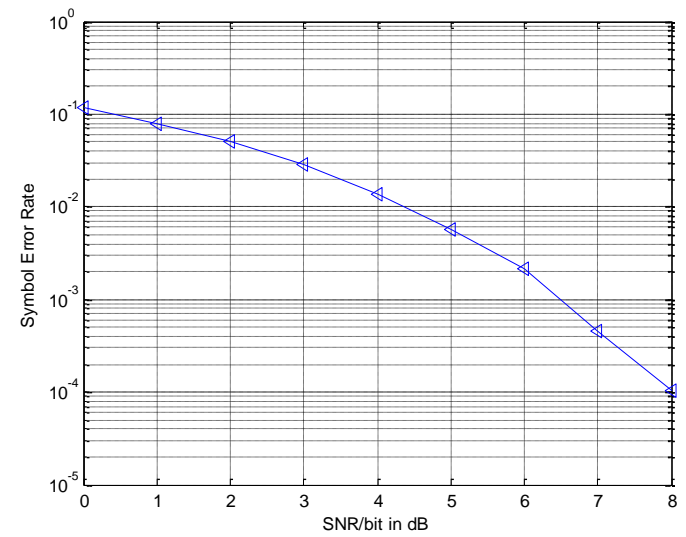

Figure 9. Symblol error rate for Gaussian pulse.

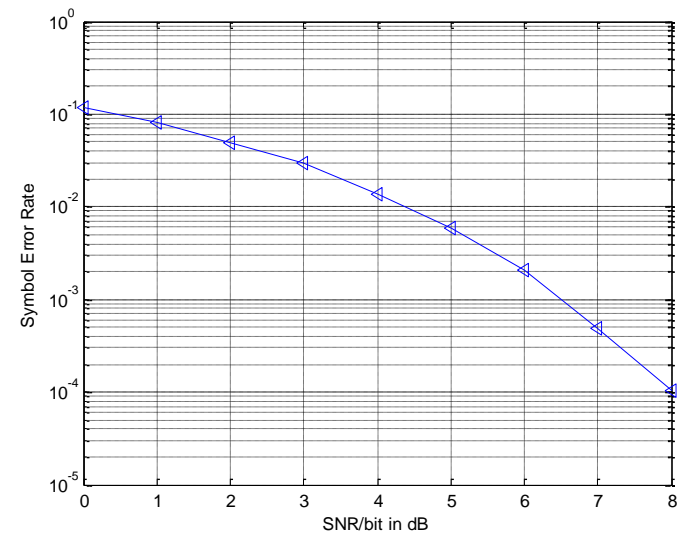

Figure 10. Symblol error rate for Hamming pulse.

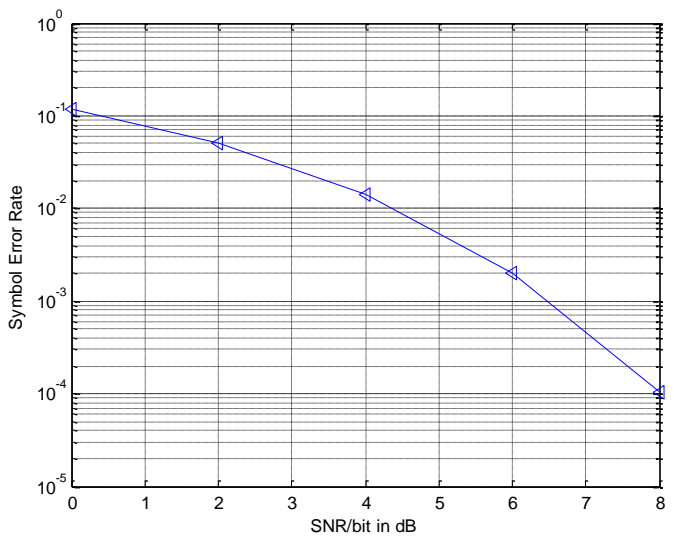

Figure 11. Symblol error rate for Hanning pulse. 


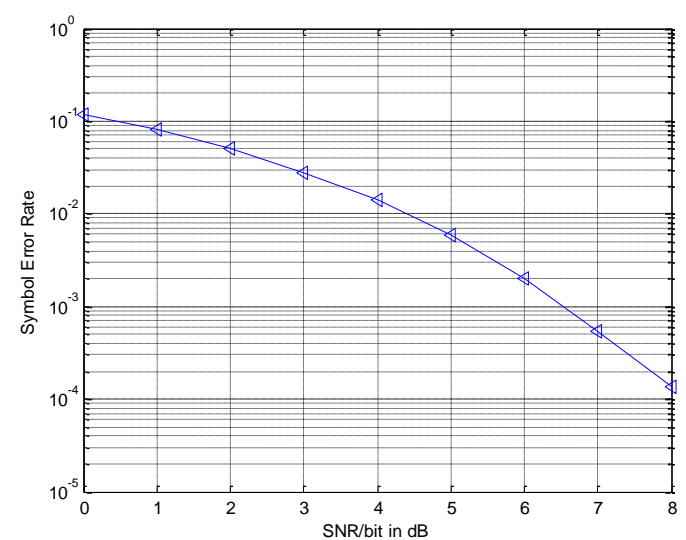

Figure 12. Symblol Error Rate for Kaiser Pulse

\section{Simulation Results and Discussion}

The data for simulated BER and theoretical BER have been evaluated for various SNR values and reported in Table-II. It is clear that as the SNR is increased the received constellation gets less affected by the noise; hence there will be fewer (less) errors.

Table 2. SNR VERSUS BER of OFDM Transmitted Signal

\begin{tabular}{|c|c|c|c|c|}
\hline $\begin{array}{c}\text { Sr. } \\
\text { No. }\end{array}$ & SNR & $\begin{array}{c}\text { BER } \\
\text { (simulated } \\
\text { ) }\end{array}$ & $\begin{array}{c}\text { BER } \\
\text { (Theoritical) }\end{array}$ & $\begin{array}{c}\text { (Sim.- } \\
\text { The.) } \\
\text { \% }\end{array}$ \\
\hline $\mathbf{1}$ & 0 & 0.1044 & 0.0786 & $2.58 \%$ \\
\hline $\mathbf{2}$ & 1 & 0.0782 & 0.0563 & $2.19 \%$ \\
\hline $\mathbf{3}$ & 2 & 0.0551 & 0.0375 & $1.76 \%$ \\
\hline $\mathbf{4}$ & 3 & 0.0360 & 0.0229 & $1.31 \%$ \\
\hline $\mathbf{5}$ & 4 & 0.0225 & 0.0125 & $1 \%$ \\
\hline $\mathbf{6}$ & 5 & 0.0120 & 0.006 & $0.6 \%$ \\
\hline $\mathbf{7}$ & 6 & 0.0058 & 0.0024 & $0.34 \%$ \\
\hline $\mathbf{8}$ & 7 & 0.0022 & 0.0008 & $0.14 \%$ \\
\hline $\mathbf{9}$ & 8 & 0.0007487 & 0.0002 & 0.0548 \\
$\%$ \\
\hline $\mathbf{1 0}$ & 9 & $\begin{array}{c}0.0001692 \\
7\end{array}$ & 0.00 & $\begin{array}{c}0.0169 \\
\%\end{array}$ \\
\hline $\mathbf{1 1}$ & 10 & $\begin{array}{c}0.0000322 \\
52\end{array}$ & 0.00 & 0.0032 \\
$\%$
\end{tabular}

However, for low values of SNR, ISI is introduced by the noise at the receiver side. This is presented in Figure- 4 to Figure- 6 which is also verified by simulated BER in TableII. It is observed from Table-II that the BER decreases as the SNR increases and its percentage error between simulated and theoretical BER value also decreases.

The bit error rate after pulse shaping the OFDM signal with different pulse shapes as mentioned earlier has been investigated and reported in tabular form Table-III. It reveals from Table-III that the BER has low value for the case of Hamming pulse at $8 \mathrm{~dB}$ SNR as compare to other pulses, indicating an improvement in the performance of an OFDM system on pulse shaping the signal with Hamming window as compared to the other pulse shapes taken into consideration. 


\section{Conclusion}

The Additive White Gaussian Noise (AWGN) corrupted the transmitted signal was transmitted and the resultant received 4-QAM constellation was compared with the original constellation. It was observed that for small SNR values the calculated error rate was quite large and ISI was produced due the relative high power of noise present but as SNR was increased the error rate was decreasing, as expected. In fact, for a SNR value greater than 8 $\mathrm{dB}$, the error was zero. This is due to the fact that the program is simulating only 68 OFDM symbols (i.e. one frame), sent one by one. As the SNR is increased the received constellation gets less affected by the noise and hence ISI becomes decreased. Thus it reveals that this work will act as the stepping stone especially in the designs of $4 \mathrm{G}$ (Generation) or future Mobile communication system which have to handle a huge quantity of data.

\section{Acknowledgment}

The first author would like to thanks G. S. Tomar for stimulating discussions, for providing a format and for critical review of this paper. The author would also like to thanks the anonymous reviewers for their valuable comments and suggestions that enhanced the quality of the paper.

\section{References}

[1] IEEE Standard 802.11a-1999: Wireless LAN MAC and PHY Specifications-High-Speed Physical Layer in the $5 \mathrm{GHz}$ Band, New York, IEEE, (2000).

[2] M. S. Alouini and A. J. Goldsmith, "A Unified Approach for Calculating Error Rates of Linearly Modulated Signals over Generalised Fading Channel," IEEE Transaction on Communications, vol. 47, no. 9, (1999), September, pp. 1324-1333.

[3] J. Zhang, L. L. Yang, X. Liu and L. Hanzo, "Inter-Carrier Interference Analysis of OFDM System Communicating over Rapidly-Fading Nakagami-m Channels," IEEE $9^{\text {th }}$ International Symposium on Spread Spctram Techniques and Applications, (2006).

[4] P. Banelli and S. Cacopardi, "Theoretical Analysis and Performance of OFDM Signals in Nonlinear AWGN Channels," IEEE Transaction on Communications, vol. 48, no. 3, (2000) March, pp. 430-438.

[5] B. B. Barrow, "Diversity combination of fading signals with unequal mean strengths," IEEE Transaction Communications System, vol. 11, (1963) March, pp. 73-78.

[6] B. R. Davis, "FM noise with fading channels and diversity," IEEE Transaction Communication Technology, vol.COM-19, (1971) December, pp. 1189-1200.

[7] H. Kang, W. Hwang and K. Kim, "Performance Analysis of the OFDM System with one tap Equalizer against the two-ray Multipath Channel," IEEE TENCON, (1999), pp. 45-48.

[8] M. Nazarathy, "Accurate evaluation of bit-error rates of optical communication systems using the GramCharlier series," IEEE Transactions on Communications, vol. 54, no. 2, (2006) February, pp. 295-301.

[9] P. K. Vitthaladevuni, M. S. Alouini and J. C. Kieffer, "Exact BER computation for cross QAM constellations," IEEE Transactions on Wireless Communications, vol. 4, no. 6, (2005) November, pp. 3039-3050.

[10] P. Kylemark, J. Ren, Y. Myslivets, N. Alic, S. Radic, Peter A. Andrekson and M. Karlsson, "Impact of pump phase-modulation on the bit-error rate in fiber-optical parametric-amplifier-based systems," IEEE Photonics Technology Letters, vol. 19, no. 2, pp. 79-81, (2007) January.

[11] C. Esli and H. Delic, "Coded OFDM With Transmitter Diversity for Digital Television Terrestrial Broadcosting," IEEE Transaction On Broadcasting, vol. 52, no. 4, (2006) December, pp. 586-596.

[12] M. Russell and G. L. Stiiber, "Interchannel interference analysis of OFDM in a mobile environment," IEEE $45^{\text {th }}$ Vehicular Technology Conference Proc., vol. 2, (1995), pp. 820-824.

[13] P. Tan and N. C. Beaulieu, "Improved BER Performance in OFDM Systems with Frequency Offset by Novel Pulse-Shaping,” IEEE Communications Society, (2004), pp. 230-236, Globecom.

[14] R. Saxena, J. K. Gautam and A. Kumar, "Correction to and Comments on-Minimum Bias Window for High Resolution Spectral Estimates,” IEEE Transaction on Information Theory, vol. 42, no. 3, (1996), pp. 1001 .

[15] R. Saxena, J. K. Gautam and A. Kumar, “On the Modified Bartlett-Hanning Windows (Family),” IEEE Transaction on Signal Processing, vol. 44, no. 8, (1996), August, pp. 2098-2102.

[16] T. Pollet, M. V. Bladel and M. Moeneclaey, "BER sensitivity of OFDM systems to carrier frequency offset and Wiener phase noise," IEEE Transaction on Communications, vol. 43, (1995) April, pp. 191193. 
[17] Y. H. Peng, Y. C. Kuo, G. R. Lee and J. H. Wen, "Performance Analysis of a New ICI-Self-CancellationScheme in OFDM Systems," IEEE Transaction on Consumer Electronics, vol. 53, no. 4, (2007) November, pp. 1333-1338.

[18] J. Armstrong, "Analysis of new and existing methods of reducing intercarrier interference due to carrier frequency offset in OFDM," IEEE Tranaction on Communications, vol. 47, (1999) March, pp. 365-369.

[19] A. Papoulis, "Probability, Random Variables, and Stochastic Processes," 2nd ed., New York: McGrawHill International Editions, (1984).

Table 3. BER of Pulse Shapes OFDM Signal for Different Values of SNR per Bits

\begin{tabular}{|c|c|c|c|c|c|c|c|}
\hline $\begin{array}{c}\text { Sr. } \\
\text { No. }\end{array}$ & $\begin{array}{c}\text { SNR } \\
(\mathbf{d B})\end{array}$ & BER OF Rect. & $\begin{array}{c}\text { BER OF } \\
\text { Blackman }\end{array}$ & BER OF Hanning & $\begin{array}{c}\text { BER OF } \\
\text { Gaussian }\end{array}$ & $\begin{array}{c}\text { BER OF } \\
\text { Hamming }\end{array}$ & $\begin{array}{c}\text { BER OF } \\
\text { Kaiser }\end{array}$ \\
\hline $\mathbf{1}$ & 0 & 0.1179 & 0.1184 & 0.1187 & 0.1186 & 0.1171 & 0.1187 \\
\hline $\mathbf{2}$ & 1 & 0.0812 & 0.07928 & 0.07984 & 0.07992 & 0.0808 & 0.08197 \\
\hline $\mathbf{3}$ & 2 & 0.05071 & 0.05026 & 0.05068 & 0.05072 & 0.04992 & 0.05132 \\
\hline $\mathbf{4}$ & 3 & 0.02879 & 0.0281 & 0.02825 & 0.02825 & 0.02923 & 0.02775 \\
\hline $\mathbf{5}$ & 4 & 0.01382 & 0.01399 & 0.01371 & 0.01375 & 0.01346 & 0.01395 \\
\hline $\mathbf{6}$ & 5 & 0.005922 & 0.006344 & 0.05775 & 0.0057930 & 0.005862 & 0.005991 \\
\hline $\mathbf{7}$ & 6 & 0.002 & 0.002 & 0.00219 & 0.002172 & 0.002052 & 0.001983 \\
\hline $\mathbf{8}$ & 7 & 0.0005172 & 0.0004827 & 0.0004741 & 0.0004569 & 0.0004913 & 0.0005431 \\
\hline $\mathbf{9}$ & 8 & $11.21 \times 10^{-5}$ & $16.38 \times 10^{-5}$ & $12.07 \times 10^{-5}$ & $10.34 \times 10^{-5}$ & $10.34 \times 10^{-5}$ & $13.79 \times 10^{-5}$ \\
\hline
\end{tabular}


International Journal of Signal Processing, Image Processing and Pattern Recognition Vol.8, No.6 (2015) 